\title{
On the evolution and enhanced relaxation of a homogeneous isotropic two-dimensional plasma in a uniform magnetic field
}

\author{
By M. J. HAGGERTY \\ Center for Statistical Mechanics and Thermodynamics, \\ The University of Texas at Austin
}

(Received 9 August 1977)

An enhancement of evolution rates in two-dimensional plasma models by a large factor containing the square root of the coupling parameter is known to occur. It is shown here to persist even when all collective effects are removed from weakcoupling calculations, under a simplified boundary condition preserving the isotropy of the system. Long-range vorticity is shown to develop. A careful treatment of time integrals allows irreversibility to be discussed with fewer ambiguities than usual with respect to limit ordering. Applications to threedimensional laboratory plasmas are tentatively suggested. Future computer simulations should determine the usefulness of the comparatively simple relationships found among moments of the pair correlation. The new effects are shown to be qualitatively similar to some found in other approaches to plasma interactions in uniform magnetic fields.

\section{Introduction}

\subsection{Enhanced classical diffusion of quiescent plasmas in uniform magnetic fields}

As plasma physicists have progressively reduced transport rates by avoiding instabilities, interest in diffusion mechanisms primarily associated with pair interactions has been renewed. Much effort has been concentrated on neoclassical effects, associated with spatial variations in the confining magnetic field (Hinton \& Hazeltine 1976). However, it has been realized that for quiescent plasmas under certain conditions, enhanced classical transport not associated with $\nabla \mathbf{B}$ effects is possible. The purpose of this paper is to present extensions and to suggest modifications for simplified kinetic theories of such plasmas. Our results are related to those of Montgomery and co-workers. For additional details concerning the general motivation and background, we refer to a recent conference review (Montgomery 1976). We were challenged to undertake further developments in the light of recent similar progress in gravitational kinetic theory (Haggerty \& Severne 1976).

In particular, two-dimensional plasmas give rise to sharply higher classical diffusion rates in velocity space than might once have been expected. Basically it is because the unperturbed guiding centres remain fixed, so random impulses 
can be persistent in mean direction and duration. Corresponding effects exist for three-dimensional plasmas in strong magnetic fields, associated with flutelike microstructures (Lee \& Liu 1973; Cheng \& Okuda 1977). Plasma transport rates can be found that differ from those classically expected not just by a modification of the Coulomb logarithm, but also by a fractional power of the plasma parameter and by powers of the gyrofrequency to plasma frequency ratios.

In the spirit of Montgomery's philosophy, in the present paper we remove from consideration all effects related to linear instabilities. We show that calculations with pair interaction terms, with long ranges and non-Markovian effects retained but with collective effects omitted, can lead to somewhat modified versions of previously derived kinetic equations describing rapid diffusion. Although not all effects are included, important indications emerge of a growth of correlational energy and vorticity. Qualitative and quantitative comparisons are then possible with computer simulations and with theoretical results taking collective effects partially into account (Krommes \& Oberman 1976 and references cited there). The most important specific kinetic-theoretical results of the present paper are found to be short and simple, and therefore useful, it is hoped, as inputs into future work.

\subsection{Perspective: possible effects of fute-like microstructures}

The earliest work on enhanced classical diffusion in quiescent plasmas in uniform magnetic fields concerns modification of the Coulomb logarithm. Sometimes the factor is replaced by the product of two logarithms of large numbers, one of the numbers being the ratio of ion to electron mass (Belyaev 1959; Silin 1963). Detailed examinations were subsequently made for a variety of initial values of parameters (Silin \& Chernyi 1969; Ichimaru \& Rosenbluth 1970; Rosenbluth \& Ichimaru 1972; Ichimaru 1973; Baldwin \& Watson 1977).

More excitement was aroused wien it was realized that further factors, differing from unity by more than a logarithmic quantity, can also arise. To start with, Taylor \& McNamara (1971) calculated that for a two-dimensional guidingcentre plasma, classical velocity diffusion (and hence spatial diffusion) scales according to $1 / B$ (Bohm-like) instead of $1 / B^{2}$. (Taylor \& McNamara's paper was discussed in detail by Montgomery (1975).)

For such a guiding-centre plasma, a 'negative temperature instability', involving clustering of like-charged particles and large-scale vortices, could be generated (Joyce \& Montgomery 1973; Edwards \& Taylor 1974).

Investigations along these lines, but without the guiding-centre approximation, were pursued by H. Okuda, J. Dawson, and co-workers in 1971-75 (see especially Okuda \& Dawson (1973)). There can be 'convective cells' leading to $1 / B$-transport even near local equilibrium, for large enough gyrofrequencies.

Following the important development of 'diffusing orbit' theories by $\mathrm{T}$. Dupree, J. Weinstock and others (see for example Voslamber (1972), Misguich \& Balescu (1975a) for recent contributions), a sophisticated analysis that tries for improved self-consistency was developed and applied to two-dimensional magnetoplasmas by Krommes \& Oberman (1976), using a hydrodynamical 
mode-coupling theory (see e.g. Baus (1977) and references cited there for the incorporation of hydrodynamic modes into plasma kinetic theory). Krommes $\&$ Oberman pointed out that the processes are essentially non-Markovian, and that a 'renormalization' is needed to calculate the transport even when the velocity distributions are equilibrium distributions. They conclude, however, that the more heuristic discussion of Okuda \& Dawson (1973) captures most of the essential features. Modes with long wavelengths, long lifetimes, and large energies are excited, and mediate the particle diffusion and transport.

However, the present paper is primarily concerned with effects arising from particle-particle interactions. It should be considered alongside the other approaches, in the future construction of a fully self-consistent theory. It is hoped that the simple results might be qualitatively valid in extrapolations.

\subsection{Kinetic theory with pair interactions dominant}

Vahala \& Montgomery (1971) made a promising first approach by developing a kinetic equation in the Landau (Fokker-Planck) approximation, for a twodimensional homogeneous plasma far from equilibrium. The plasma consists of a single species of rods of charge $q$ aligned along a uniform magnetic field, with a neutralizing uniform background charge. No long-range divergence difficulty occurred (when $B$ is not small). This case happens to be particularly well adapted to the philosophy of their group of ignoring the effects of excited modes associated with linear instabilities, as a starting point in describing plasmas that are quiescent on kinetic time scales. As a practical matter, it cannot be applied unless the time scales are not strictly separated, the coupling not being strictly weak, so that the discreteness effects can have a chance to dominate the collective effects (Sandri 1963; Montgomery 1967; see also Radin, Kritz \& Sandri 1967; Radin 1972). Because of the intrinsic non-Markovian behaviour, some approximations had to be made and a cut-off introduced. They chose one based on Taylor \& McNamara's (1971) diffusion process. The related discussion was updated in Hsu, Montgomery \& Joyce (1974a) and Montgomery (1976), and the results tested by computer simulations by Hsu, Joyce \& Montgomery (1974b) to their satisfaction.

In the meantime, the Balescu-Lenard limit (Vahala 1972) had been yielding some puzzling and seemingly inappropriate results. We hope to discuss them in a future work; superficially, they are associated with a lack of linear Landau damping of the (high-frequency) Bernstein modes. To the extent that the puzzling results are not verified by simulation, this challenging current situation has the advantage of increasing one's confidence in the Landau form of the weakcoupling kinetic theory as a first approximation, for single-species two-dimensional plasmas of intermediate coupling strength.

Our approach is further to refine the weak-coupling theory by removing the cut-off on the duration of the pair interactions. Instead we introduce an arbitrarily large long-range cut-off $\kappa^{-1}$, appropriate for a finite system without effects associated with special boundaries. The motive is to isolate the collective effects by removing them entirely from the weak-coupling description, so as to get 
another standard for comparison with computer simulations. In other words, within Montgomery's basic approach, and in view of the still incomplete but more sophisticated theories of collective effects mentioned in $\S 1.2$, it seems wise to find a description incorporating only pair interactions. The results we find preserve considerable simplicity. Some order-of-limit ambiguities resolve themselves quite naturally for this model. Certain features expected for a more complete kinetic theory emerge in a form that is perhaps rudimentary but qualitatively plausible.

In particular, we find that the evolution rates present the same anomalous dependence on the plasma parameter as was found in previous calculations, but not the same dependence on the gyrofrequencies. Moreover, by going beyond the approximations of Vahala \& Montgomery, we find long-range effects associated with growing correlational energy and vorticity.

\subsection{Order of presentation}

After writing the equations pertaining to the starting point, we proceed in $\S 3$ to an examination of the weak-coupling kinetic equation. $\$ 3.2$ involves a treatment at the level of approximation used by Montgomery and co-workers, only without their cut-off in the time integral. Comparisons are made between the results of the two versions of the theory. In one limiting case we find that the kinetic energy density is not approximately conserved, just as in infinite gravitational systems.

It is convenient to work also with the corresponding correlational (potential) energy density ( $\$ 4.1$ and 4.2). Fewer limits than usual need be taken, and equations (4.6) and (4.13), with figure 1, show that qualitatively different behaviours are connected as a family. Irreversibility is then discussed briefly, from a somewhat unusual point of view (\$4.3).

In $\S \S 4.4$ and 5 , we introduce a less stringent approximation, giving in some cases the dominant growth for moments of the correlation function. This 'quasistroboscopic approximation' retains part of the variation of the velocity disdistribution during each gyroperiod. It predicts a long-lived retention of memory of the initial distribution, as well as simple relations between various moments that can later be tested by computer simulation. In particlar, vorticity having a large characteristic length scale is predicted to develop. Its amplitude is predicted to be proportional to that of a component of the correlational energy density whose characteristic length scale is not as large.

Applications are discussed briefly among the subsequent remarks in $\S \S 6$ and 7.

\section{Basic considerations}

\subsection{Physical model}

The particles, $N$ in number, are very long rods of length $l$, charge $q$, and mass $m$, aligned along a uniform magnetic field $\mathbf{B}=B \hat{z}$. They are distributed with statistical homogeneity in a cylindrical volume of cross-sectional area $L^{2}$, containing a neutralizing background charge density $(-q / l) n_{0}$, where $n_{0}=N / L^{2}$ 
(cf. Hsu et al. 1974a). The one-particle distribution function is isotropic in velocity space:

$$
f(i)=n_{0} \varphi\left(v_{i}, t\right)
$$

No special boundaries are introduced that would give an evolution breaking the symmetries of homogeneity and isotropy. Instead, the Coulomb potential is cut off at a large distance $\kappa^{-1}$. We have in mind the identification $\kappa^{-1} \approx L$, but do not use this relationship; instead we construct the pair potential according to equilibrium shielding theory (Abraham-Shrauner 1969; cf. Edwards \& Taylor 1974):

$$
\mathbf{F}_{i j}=-\nabla V^{i j}=\frac{2 q^{2} \kappa}{m l x_{i j}} K_{1}\left(\kappa x_{i j}\right) \mathbf{x}_{i j}
$$

is the partial acceleration of $\operatorname{rod} i$ at $\mathbf{x}_{i}=\left(x_{i}, y_{i}\right)$ due to the presence of rod $j$ at $\mathbf{x}_{j}$, where

$$
V^{i j}=V^{i j}\left(x_{i j}\right)=V^{i j}\left(\left|\mathbf{x}_{i}-\mathbf{x}_{j}\right|\right)=\frac{2 q^{2}}{m l} K_{0}\left(\kappa x_{i j}\right)
$$

Here, $K_{0}$ and $K_{1}$ are Bessel functions. The limit $\kappa \rightarrow 0$ gives the logarithmic Coulomb potential between rods, and may be taken at any of several points in the calculation. Also, there is no short-range divergence difficulty.

Moreover, we assume that there is an initial time $t_{0}$ for which there are no pair correlations except those already modelled through the pair potential. In other words, the correlation function

$$
g(i, j)=f_{2}(i, j)-f(i) f(j)=n_{0}^{2} P_{i j}
$$

is zero at that time:

$$
g\left(t=t_{0}\right)=0 \text {. }
$$

It can be said that we model a finite system by an infinite system with a cut-off potential. Because of the finite diameter, we limit the length scale of developing pair correlations. The limit is associated either with what amounts to a simple isotropy-preserving 'boundary condition' if $\kappa^{-1} \approx L$, or with a shielding by a mobile species whose discreteness effects are not otherwise taken into account. No 'particle' (no rod) is allowed to feel the effects of being close to the edge of the cylinder; they all sense a vacuum or a shield at ranges exceeding $\kappa^{-1}$ in order of magnitude.

\subsection{Weak-coupling approximation}

The gyrations of the particles in the field $B$, in the absence of accelerations (2.2) due to pair interactions, would leave the relative separations bounded. There is a constant recurrence time $2 \pi /|\Omega|$, where $\Omega$ is the gyrofrequency

$$
\Omega=-(q / m c)|\mathbf{B}| \text {. }
$$

Usually one calculates impulses due to weak pair interactions by integrating along the corresponding unperturbed orbits. But this cannot be an accurate approximation unless time scales are allowed such that the impulses could change the velocity distribution. The usual physical picture breaks down; we 
should think of integrating evolving distributions of particles around angles rather than integrating along single-particle trajectories. The description is said to be essentially non-Markovian. Since we are not including the energetic modes discussed in \$1.2, nor the Taylor-McNamara diffusion mechanism, our range for the time integrals is simply the elapsed time itself, $t-t_{0}$. Thus it is predicted that some memory of the initial conditions at $t_{0}$ is long-lasting, and is retained on kinetic time scales. This feature is one item whose limits of approximate validity should be checked by simulation.

Other features are present that are independent of the choice of cut-off, and agree with results of Abraham-Shrauner (1969) and Vahala \& Montgomery (1971) in the appropriate limits. While the validity of our model will depend on the parameters, within the model all gyrofrequencies can be treated except those near the relaxation time scale. (In the exceptional region, complications similar to those for finite systems with recurrence times comparable to the relaxation times inevitably arise, to make the evolution more difficult to calculate (e.g. Davidson \& Kozak 1970).) We made it a point to assume no scaling relationship between any two of the gyroperiod, the inverse plasma frequency, and the time for which a distance $\kappa^{-1}$ might typically be traversed by a particle; the interested reader would find the integrals easier to check by taking limiting cases.

Specifically, the starting point is the usual one for weak coupling, namely the first two equations of the BBGKY hierarchy, with collective terms and triplet correlations ignored:

$$
\begin{gathered}
\frac{\partial f(1)}{\partial t}=\iint d \mathbf{v}_{2} \iint d \mathbf{x}_{2} \Theta_{12} g(1,2), \\
\frac{\partial g(1,2)}{\partial t}+\left(\mathbf{v}_{1} \cdot \nabla_{1}+\mathbf{v}_{2} \cdot \nabla_{2}\right) g+\Omega\left(\frac{\partial}{\partial \phi_{1}}+\frac{\partial}{\partial \phi_{2}}\right) g=\Theta_{12} f(1) f(2),
\end{gathered}
$$

with the initial condition (2.5). Here, $\phi_{i}$ is the angle between the velocity $\mathbf{v}_{i}=v_{i x} \hat{x}+v_{i \nu} \hat{y}$ and a fixed direction $\hat{x}$ normal to $\mathbf{B}=B \hat{z}$; also,

where $\mathbf{v}_{i j}=\mathbf{v}_{i}-\mathbf{v}_{j}$.

$$
\begin{gathered}
\Theta_{i j}=\left(\nabla_{i} V^{i j}\right) \cdot \partial_{i j}, \\
\partial_{i j}=\frac{\partial}{\partial \mathbf{v}_{i}}-\frac{\partial}{\partial \mathbf{v}_{j}}=\left.2 \frac{\partial}{\partial \mathbf{v}_{i j}}\right|_{\nabla_{i}+\mathrm{v}_{j}},
\end{gathered}
$$

\subsection{Evolution of the pair correlation function}

In considering the time-integration of (2.8), (2.5), we first notice that the two operators $\left(\mathbf{v}_{1} \cdot \nabla_{1}+v_{2} \cdot \nabla_{2}\right)$ and $\Omega\left(\partial / \partial \phi_{1}+\partial / \partial \phi_{2}\right)$ do not commute with each other, nor does their commutator itself commute with both operators. For a very readable account of how one should 'dress' one operator with the other, we refer to Misguich \& Balescu (1975b). The result is a time integral containing explicit displacement operators:

$$
g(t)=\int_{0}^{t-t_{0}} d \tau U(t, t-\tau) \Theta_{12} f(1, t-\tau) f(2, t-\tau)
$$




$$
\begin{aligned}
& U(t, t-\tau) \\
& =\exp \left(-\left[\mathbf{v}_{1} \cdot \mathbf{G}^{\dagger}(\tau) \cdot \nabla_{1}+\mathbf{v}_{2} \cdot \mathbf{G}^{+}(\tau) \cdot \nabla_{2}\right]\right) \exp \left(-\tau \Omega\left[\partial / \partial \phi_{1}+\partial / \partial \phi_{2}\right]\right), \\
& \mathbf{G}(\tau)=\int_{0}^{\tau} d \tau^{\prime} \mathbf{R}\left(\tau^{\prime}\right)=\frac{1}{\Omega}\left(\begin{array}{cc}
\sin \Omega \tau & 1-\cos \Omega \tau \\
-(1-\cos \Omega \tau) & \sin \Omega \tau
\end{array}\right) \\
& =-\mathbf{G}^{+}(-\tau) \text {, } \\
& \mathbf{R}(\tau)=\left(\begin{array}{cc}
\cos \Omega \tau & \sin \Omega \tau \\
-\sin \Omega \tau & \cos \Omega \tau
\end{array}\right)=\mathbf{R}^{\dagger}(-\tau)
\end{aligned}
$$

Equation (2.7), with (2.11) substituted, will yield a kinetic equation containing not precisely a two-time force-autocorrelation function, but rather a similar expression with additional memory effects owing to the long duration of the pair interactions.

\section{Kinetic equation}

3.1. Non-Markovian kinetic equation

Explicitly, the kinetic equation just derived for the velocity distribution $\varphi(|\mathbf{v}|, t)$ is

$$
\begin{aligned}
\partial \varphi\left(v_{1}, t\right) / \partial t=n_{0} \int & d \mathbf{v}_{2} d \mathbf{x}_{2}\left(\nabla_{1} V^{12}\right) \cdot \partial_{12} \int_{0}^{t-t_{0}} d \tau \\
& {\left[\nabla_{1} V^{12}\right]_{\left\{\mathbf{x}_{i} \rightarrow \mathbf{x}_{i}-\nabla_{i} \cdot \mathbf{G} t(\tau)\right\}} \cdot \mathbf{R}(\tau) \cdot \partial_{12} \varphi\left(v_{1}, t-\tau\right) \varphi\left(v_{2}, t-\tau\right) . }
\end{aligned}
$$

Working out the integration with respect to $x_{2}$ (by taking Fourier transforms and differentiating with respect to parameters), we get

$$
\begin{aligned}
& \frac{\partial \varphi\left(v_{1}, t\right)}{\partial t}=C^{\prime} \int d \mathbf{v}_{2} \int_{0}^{t-\tau} d \tau \partial_{12} \cdot\left[K_{0}\left(Y_{\tau}\right)\right.\left.-K_{1}\left(Y_{\tau}\right) \frac{\mathbf{Y}_{\tau} \mathbf{Y}_{\tau}}{Y_{\tau}}\right] \\
& \cdot \mathbf{R}(\tau) \cdot \partial_{12} \varphi\left(v_{1}, t-\tau\right) \varphi\left(v_{2}, t-\tau\right)
\end{aligned}
$$

where

$$
\begin{aligned}
& \mathbf{Y}_{\tau}=\kappa \mathbf{G}(\tau) \cdot \mathbf{v}_{12}=\kappa \mathbf{v}_{12} \cdot \mathbf{G}^{\dagger}(\tau) \\
& C^{\prime}=\frac{4 \pi q^{4} n_{0}}{m^{2} l^{2}}=\frac{C}{\rho} \\
& C=\frac{4 \pi q^{4} n_{0}^{2}}{m l^{3}}, \quad \rho=\frac{m n_{0}}{l}=\frac{m N}{l L^{2}}
\end{aligned}
$$

and

$$
\mathbf{I}=\left(\begin{array}{ll}
1 & 0 \\
0 & 1
\end{array}\right) .
$$

Note that

$$
\mathbf{Y}_{\tau} \cdot \mathbf{R}(\tau)=\kappa \mathbf{v}_{12} \cdot \mathbf{G}(\tau)
$$

\section{(a) Evolution of $\varphi$}

\subsection{Stroboscopic approximation}

Montgomery (1975) has suggested using a 'stroboscopic approximation' (cf. Minorsky 1962) as a first approximation for treating the integral with respect 
to $\tau$. To the extent that the isotropic velocity distribution $\varphi(|\mathbf{v}|)$ changes slowly on the time scale of a gyroperiod $2 \pi /|\Omega|$, we may write

$$
\begin{aligned}
& \varphi\left(v_{1}, \tau-\pi|\Omega|^{-1}[2 \mu-1]-\delta \tau\right) \varphi\left(v_{2}, t-\pi|\Omega|^{-1}[2 \mu-1]-\delta \tau\right) \\
& \quad \approx \varphi\left(v_{1}, t-\pi|\Omega|^{-1}[2 \mu-1]\right) \varphi\left(v_{2}, t-\pi|\Omega|^{-1}[2 \mu-1]\right), \quad \mu=1,2,3, \ldots
\end{aligned}
$$

whenever

$$
|\delta \tau| \leqslant \pi /|\Omega| \text {. }
$$

The first correction to (3.7), to be called 'quasi-stroboscopic,' will be introduced in $\$ 4.4$.

Moreover, in order to extract the secular part of the evolution, we take

$$
t-t_{0} \approx 2 \pi \mu_{1} /|\Omega|
$$

for some integer $\mu_{1}$. Then (3.2) becomes

$$
\partial \varphi\left(v_{1}, t\right) / \partial t \approx-\int d \mathbf{v}_{2} \partial_{12} \cdot \mathbf{Q} \cdot \partial_{12}\left\langle\varphi\left(v_{1}\right) \varphi\left(v_{2}\right)\right\rangle_{t},
$$

where $\quad \mathbf{Q}=-\frac{\left(t-t_{0}\right)|\Omega| C^{\prime}}{2 \pi} \int_{0}^{2 \pi /|\Omega|} d \tau\left[K_{0}\left(Y_{\tau}\right) \mathbf{l}-K_{1}\left(Y_{\tau}\right) \frac{\mathbf{Y}_{\tau} \mathbf{Y}_{\tau}}{Y_{\tau}}\right] \cdot \mathbf{R}(\tau)$,

$$
\begin{aligned}
& \left\langle\varphi\left(v_{1}\right) \varphi\left(v_{2}\right)\right\rangle_{t}=\frac{1}{\mu_{1}} \sum_{\mu=1}^{\mu_{1}} \varphi\left(v_{1}, l-\pi|\Omega|^{-1}[2 \mu-1]\right) \varphi\left(v_{2}, t-\pi|\Omega|^{-1}[2 \mu-1]\right), \\
& \left\langle\varphi\left(v_{1}\right) \varphi\left(v_{2}\right)\right\rangle_{t} \approx\left(t-t_{0}\right)^{-1} \int_{0}^{t-t_{0}} d \tau \varphi \varphi(t-\tau) .
\end{aligned}
$$

Let

$$
a=a_{12}=\kappa v_{12} /|\Omega|,
$$

so that

$$
Y_{\tau}=2 a|\sin (\Omega \tau / 2)|=[2(1-\cos \Omega \tau)]^{\frac{1}{2}} a .
$$

After some manipulations, using in particular the standard formula

$$
\int_{0}^{\pi / 2} d \theta(\cos 2 \theta) K_{0}(2 a \cos \theta)=-\frac{\pi}{2} I_{1}(a) K_{1}(a)
$$

and its derivatives

$$
\begin{aligned}
\int_{0}^{\pi / 2} d \theta(\cos \theta) K_{1}(2 a \cos \theta) & =\frac{\pi}{2}\left[I_{0}(a) K_{1}(a)-\frac{1}{2 a}\right], \\
\int_{0}^{\pi / 2} d \theta(\cos 3 \theta) K_{1}(2 a \cos \theta) & =-\frac{\pi}{2}\left[I_{1}(a) K_{2}(a)-\frac{1}{2 a}\right],
\end{aligned}
$$

with

$$
\begin{gathered}
I_{n}(a) K_{n+1}(a)+I_{n+1}(a) K_{n}(a)=1 / a, \\
K_{2}(a)=K_{0}(a)+(2 / a) K_{1}(a), \quad \text { etc., }
\end{gathered}
$$

we find that

$$
\begin{aligned}
\mathbf{Q}=-\left(t-t_{0}\right) C^{\prime} M^{(1)}(a)\left[\mathrm{I}-\hat{v}_{12} \hat{v}_{12}\right], \quad \hat{v}_{12}=\mathrm{v}_{12} / v_{12}, \\
\mathbf{Q}=-\left(t-t_{0}\right) \frac{4 \pi q^{4} n_{0}}{m^{2} l^{2} v_{12}^{2}} M^{(1)}\left(\frac{\kappa v_{12}}{|\Omega|}\right)\left[\mathbf{v}_{12} \times \hat{z}\right]\left[\mathbf{v}_{12} \times \hat{z}\right],
\end{aligned}
$$

or 
where

$$
\begin{aligned}
M^{(1)}(a) & =a I_{0}(a) K_{1}(a)-a I_{1}(a) K_{0}(a) \\
& =1-2 a I_{1}(a) K_{0}(a) .
\end{aligned}
$$

For $a=\{0,0 \cdot 2,1,3,10\}$,

$$
M^{(1)}(a)=\{1,0.929,0.524,0 \cdot 176,0.035\} .
$$

\section{(b) Comparison with a previous result: limit $\kappa \rightarrow 0$}

In the limit $\kappa \rightarrow 0$,

$$
M^{(\mathbf{1})}(a) \rightarrow 1,
$$

and (3.10), (3.22) reduce to the Vahala-Montgomery kinetic equation ((31) of Vahala \& Montgomery 1971), except with $T_{0} f_{1} f_{1}$ replaced by $\left(t-t_{0}\right)\left\langle f_{1} f_{1}\right\rangle_{t}$, i.e. without a cut-off $T_{0}$. The behaviour of $M^{(1)}(a)$, according to (3.24), is plausible in the light of the finite effective range of the interactions in their approximations (cf. (208) of Montgomery 1975).

Vahala \& Montgomery's choice for the cut-off $T_{0}$ was dictated by heuristic arguments. One of its pleasing features is here pointed out for the first time: the time scale of evolution that results, proportional to the square root of the product of a long and a short time scale, is a natural one. We call it a hybrid time scale (Haggerty \& Severne 1976). It is natural on the basis of time-scaling arguments, since the standard argument for the absence of such evolution (Montgomery 1967 , p. 64) breaks down when there is no asymptotic separation of the unperturbed orbits. Indeed, we can point out that the present version of the theory (without any $T_{0}$ ) preserves the property, as is clear upon dividing (3.10) by $\left(t-t_{0}\right)$ and writing the resulting left-hand side in the form $\partial \varphi / \partial\left[\frac{1}{2}\left(t-t_{0}\right)^{2}\right]$. The calculation of the order of magnitude of the time scale would involve taking the square root of the constant $C^{\prime}$ of (3.21) or (3.22). Figure 4 of Hsu et al. (1974b) shows the validity of the property in the simulation models as well, at least for one moment, namely Boltzmann's $H$.

Let us note that the resulting approximately-Gaussian time dependence $\left[\propto \exp \left(-\alpha\left[t-t_{0}\right]^{2}\right)\right]$ has previously arisen in the theory and simulation of twodimensional magnetoplasmas (Weinstock 1976; Okuda \& Dawson 1973), but in the context of time-correlation functions. The explanation in terms of 'inertial' motion would be similar in the two contexts, at least for early times.

The condition $g\left(t=t_{0}\right)=0$ implies that $\left\{\partial \varphi / \partial t=0\right.$ at $\left.t=t_{0}\right\}$ (cf. (2.7)). Therefore when the simulations fail to agree (cf. figure 2, Hsu et al., 1974b), it shows that there is effectively a spurious initial correlation or a strong short-range interaction. We would thus argue that the result $\left\{d H / d t<0\right.$ at $\left.t=t_{0}\right\}$ shown both by the simulation and by the kinetic equation with a cut-off $T_{0}$ does not by itself argue against our version with the factor $\left(t-t_{0}\right)$ on the right-hand side; the cut-off version cannot be accurate for $t<t_{0}+T_{0}$.

Somewhat more serious is the question of the preservation of the condition of non-negative probabilities: $\varphi(v, t) \geqslant 0$. If $(3.13),(3.21)$ is substituted into (3.10), the factors $\left(t-t_{0}\right)$ cancelled, and a second time derivative taken (after a substitution $\left.t^{\prime}=t-\tau\right)$, one gets an equation suggesting oscillations. Or, to approach it another way: to the extent that $d H / d t$ is driven by historical rather 
than current values of $\varphi\left(v_{1}\right) \varphi\left(v_{2}\right)$, one can easily imagine that there could be an overshoot of predicted intermediate values of $H$ that would be unphysical. And even if they are not unphysical, the $H$-theorem in its strictest sense would not be expected to be valid (not even within the stroboscopic approximation).

In response, we can argue that most non-Markovian kinetic equations have the same property: the proof of $\varphi(t) \geqslant 0$ is not immediate, and does not necessarily exist. $B$ ut it has not been shown to lead to major inaccuracies in the prediction of important properties of the evolution. We know that values of $\varphi$ given by the theory are approximate; if some happen to be negative, it doesn't necessarily mean that the results as a whole will give poor predictions. Some oscillations are observed in some of the simulations.

Therefore, despite some reservations, we propose that models without a cut-off $T_{0}$ be chosen as the best standards for comparison of theory and computer experiments, until collective effects have been introduced on a fully self-consistent basis. An example of a feature depending on $T_{0}$ would be the dependence of the evolution rate on the magnetic field strength. Because of (3.21), (3.25), our result suggests a plateau in the $B$-dependence not unlike the regions near the minima of the curves in figure 3 of Hsu et al. (1974b). While their figure 4 confirms to a good approximation a feature of both versions, the presently available simulation results do not appear to discriminate unambiguously between one version and the other. (See also Montgomery (1976), § 3.1, referring to a similar modification suggested by the work of Okuda \& Dawson (1973).)

Finally we point out the unusual importance of the assumption of initial statistical homogeneity. Hybrid-time-scale evolution is possible only when short time-scale effects could in principle be long-lasting, rather than leading to an immediate polarization (or instability). In the intermediate-coupling extrapolation, the matter can to some extent be resolved by adjusting the choice of initial ensemble (Haggerty \& Severne 1976), but more so for gravitational systems than for plasmas.

\section{(c) Kinetic energy density}

It is easily verified that there is no secular evolution of the kinetic energy density

$$
\mathscr{E}^{\mathrm{K}}(t)=\frac{1}{2} \rho \int d \mathbf{v}_{1} v_{1}^{2} \varphi\left(v_{1}, t\right)
$$

in the stroboscopic approximation:

$$
d \mathscr{E}^{\mathrm{K}}(t) / d t=0 \quad(\text { for all } \kappa) .
$$

It makes sense that there should be no secular evolution of kinetic energy on the hybrid time scale, in the stroboscopic approximation, because the corresponding potential energy could not grow like $C^{\prime}\left(t-t_{0}\right)^{2}$; it is limited to a growth proportional to the first power of $\left(t-t_{0}\right)$ at most, since the underlying correlation function (2.11) has a periodic kernel in that approximation. Also, it is easy to verify that the total energy is conserved at the level of the starting point (2.7), (2.8). 


\subsection{Limit of weak magnetic fields}

As previously pointed out, the long-range cut-off $\kappa^{-1}$ is not necessarily identified with a thermal-velocity to plasma-frequency ratio, so the relaxation time is not necessarily larger than either $|\Omega|^{-1}$ or $\left(\kappa\left\langle v_{12}\right\rangle\right)^{-1}$. In particular, since $|\Omega|\left(t-t_{0}\right)$ need not be large, the choice (3.9) of 'secular part' is not always appropriate, at least in the mathematical (strictly two-dimensional) model appropriate for simulation if not laboratory experiments.

(a) Screened rods without gyrations

We take

$$
1 / \kappa v_{12} \ll t-t_{0} \ll 1 /|\Omega|,
$$

to make contact with Abraham-Shrauner (1969). Now there are no recurrences, so one expects a Markovianization to be appropriate. Thus the $\tau$-dependence of $\varphi \varphi(t-\tau)$ can again be dropped: in (3.2),

$$
\varphi \varphi(t-\tau) \rightarrow \varphi \varphi(t),
$$

and also $\quad Y_{\tau}=2 a|\sin (\Omega \tau / 2)| \approx a|\Omega| \tau=\kappa v_{12} \tau, \quad \mathbf{R}(\tau) \approx \mathrm{I}$.

We must calculate

$$
\mathbf{Q}^{\Omega=0}=-C^{\prime} \int_{0}^{\kappa v_{12}\left(t-t_{0}\right)} \frac{d Y}{\kappa v_{12}}\left[K_{0}(Y) \mid-Y K_{1}(Y) \frac{\mathbf{v}_{12} \mathbf{v}_{12}}{v_{12}^{2}}\right] .
$$

With $\kappa v_{12}\left(t-t_{0}\right) \gg 1$, and since

we easily find that

$$
\int_{0}^{\infty} K_{0}(x) d x=\pi / 2
$$

$$
\mathbf{Q}^{\Omega=0}=-\frac{\pi C^{\prime}}{2 \kappa v_{12}}\left(1-\frac{\mathbf{v}_{12} \mathbf{v}_{12}}{v_{12}^{2}}\right) \quad[\kappa \text { large }]
$$

and

$$
\partial \varphi\left(v_{1}, t\right) / \partial t=-\int d \mathbf{v}_{2} \partial_{12} \cdot \mathbf{Q}^{\Omega=0} \cdot \partial_{12} \varphi\left(v_{1}, t\right) \varphi\left(v_{2}, t\right)
$$

in agreement with Abraham-Shrauner's result.

Moreover, we have a qualitative agreement between (3.32) and (3.21), except for the loss of proportionality of the relaxation time to the square root of the coupling parameter. In particular,

$$
d \mathscr{E} \mathrm{K} / d t=0
$$

(b) Unscreened rods without gyrations

In (3.31), let us allow $\kappa$ to decrease until

Then we can use

$$
\kappa\left\langle v_{12}\right\rangle\left(t-t_{0}\right) \ll 1 .
$$

$$
K_{0}(x)=\ln \frac{2 e^{-\gamma}}{x}+\frac{x^{2}}{4} \ln \frac{2 e^{1-\gamma}}{x}+O\left(x^{4} \log \frac{1}{x}\right)
$$

and

$$
K_{1}(x)=\frac{1}{x}-\frac{x}{2} \ln \frac{2 e^{\frac{1}{2}-\gamma}}{x}+O\left(x^{3} \log \frac{1}{x}\right)
$$


$\gamma=0.5772$ (Euler's constant), to arrive at results quite different from the ones for $\kappa$ large:

$$
\mathbf{Q}_{\kappa \rightarrow 0}^{\Omega=0} \approx-\left(t-t_{0}\right) C^{\prime}\left\{\left[\ln \frac{2 e^{1-\gamma}}{\kappa v_{12}\left(t-t_{0}\right)}\right] I-\frac{\mathbf{v}_{12} \mathbf{v}_{12}}{v_{12}^{2}}\right\} .
$$

Note that one cannot arrive at (3.38) from (3.21), (3.23), even by formally assigning varying values to the indeterminate quantity $\left\{a=\left(\kappa v_{12} /|\Omega|\right), \kappa \rightarrow 0\right.$, $\Omega \rightarrow 0\}$.

Equations (3.33), (3.38) give an evolution for the kinetic energy density

$$
\left.\frac{d \mathscr{E}^{\mathrm{K}}}{d t}\right|_{\kappa \rightarrow 0} ^{\Omega=0} \approx 2\left(t-t_{0}\right) C \int d \mathbf{v}_{1} d \mathbf{v}_{2} \varphi\left(v_{1}, t\right) \varphi\left(v_{2}, t\right) \ln \frac{2 e^{-\gamma-\frac{1}{2}}}{\kappa v_{12}\left(t-t_{0}\right)}
$$

that is quite similar to that found for gravitational systems (compare for example (30) of Ostriker \& Davidsen 1968). It has a sign (cf. (3.35)):

$$
\left(d \mathscr{E}^{K} / d t\right)_{k \rightarrow 0}^{\Omega=0}>0
$$

since the logarithm is negative only where $\varphi \varphi$ is small. The rate is insensitive to the details of the shape of $\varphi \varphi$, thus justifying a posteriori the relatively cavalier treatment of its $\tau$-dependence implied by (3.35), (3.38) (cf. the discussion of a related ansatz in Haggerty \& Severne 1976).

The physical interpretation is that there is a net average increase in speeds due to repulsions between pairs with low relative velocities, resulting in negative spatial correlations ('anticorrelations'). This goes on, according to the prediction, until either $|\Omega|\left(t-t_{0}\right)$ or $\kappa\left\langle v_{12}\right\rangle\left(t-t_{0}\right)$ is not small, or until the weakcoupling approximation breaks down. We will re-examine the region

$$
|\Omega|\left(t-t_{0}\right)>1
$$

in connexion with moments of the correlation function.

\section{Correlational energy density}

\subsection{General expression}

The correlational energy density

$$
\mathscr{E}^{V}=\mathscr{E}_{\mathrm{corr}}=\frac{1}{2}(\rho / N) \iint d \mathbf{v}_{1} \iint d \mathbf{v}_{2} \iint d \mathbf{x}_{1} \iint d \mathbf{x}_{2} V^{12} g(1,2)
$$

may be calculated from (2.11), (2.3), and the spatial integration performed using Fourier transforms. The result is

$$
\mathscr{E} V=\frac{1}{2} C \int d \mathbf{v}_{1} d \mathbf{v}_{2} \mathbf{v}_{12} \cdot \int_{0}^{t-t_{0}} d \tau K_{0}\left(Y_{\tau}\right) \mathbf{G}(\tau) \cdot \partial_{12} \varphi\left(v_{1}, t-\tau\right) \varphi\left(v_{2}, t-\tau\right)
$$

(See (2.10), (2.13), (3.3) and (3.5) for definitions of symbols.) It is to be remembered that this is valid for the initial condition $g\left(t_{0}\right)=0$. We shall examine the complications arising from the $\tau$-dependence of $\varphi \varphi$ in $\$ 4.4$ below. 


\subsection{Stroboscopic, short-time, or Markovian approximations}

In several circumstances, it will be appropriate to neglect the $\tau$-dependence of $\varphi \varphi$, and to use a tensor $\mathrm{J}\left(v_{12}, t\right)$ defined by

$$
\mathbf{J}=\int_{0}^{t-t_{0}} d \tau K_{0}\left(Y_{\tau}\right) \mathbf{G}(\tau)
$$

(not a current). The diagonal elements of $J$ are the important ones:

$$
\operatorname{Tr} \mathbf{J}=2 J_{x x}=2 J_{y y}=\frac{2}{\kappa^{2} v_{12}^{2}}-\frac{4\left|\sin \frac{1}{2} \Omega\left(t-t_{0}\right)\right|}{\kappa v_{12}|\Omega|} K_{1}\left(\frac{2 \kappa v_{12}}{|\Omega|}\left|\sin \frac{1}{2} \Omega\left(t-t_{0}\right)\right|\right),
$$

because

$$
\mathscr{E}^{\nabla}=\frac{1}{2} C \int d \mathbf{v}_{1} d \mathbf{v}_{2} \mathbf{v}_{12} \cdot \mathbf{J} \cdot \partial_{12} \varphi \varphi,
$$

or, since $J_{x x}=J_{y y}$ and $J_{x y}=-J_{y x}$,

$$
\begin{aligned}
\mathscr{E}^{V}=-C \int d \mathbf{v}_{1} d \mathbf{v}_{2} \varphi\left(v_{1}\right) \varphi\left(v_{2}\right)\left[(\operatorname{Tr} \mathbf{J})+\frac{1}{2} v_{12}\left(\partial / \partial v_{12}\right)(\operatorname{Tr} \mathbf{J})\right] \\
-\pi C\left\{\int d \mathbf{v}[\varphi(v)]^{2}\right\}\left\{\lim _{\left(\boldsymbol{v}_{\mathbf{1}} \mid\left\langle\mathbf{v}_{12}\right\rangle\right) \rightarrow 0} v_{12}^{2}(\operatorname{Tr} \mathrm{J})\right\} .
\end{aligned}
$$

When $|\Omega|\left(t-t_{0}\right) \gg 1$, these expressions will be valid under the stroboscopic approximation (3.7), if $\varphi \varphi$ is replaced by $\langle\varphi \varphi\rangle_{t}$ defined by (3.12). Another 'large $\Omega$ ' approximation is

in which case

$$
a=\kappa v_{12} /|\Omega| \ll 1 \text {, }
$$

$$
\operatorname{Tr} \mathbf{J} \approx \frac{4}{\Omega^{2}}\left[\sin ^{2} \frac{1}{2} \Omega\left(t-t_{0}\right)\right] \ln \left[\frac{|\Omega| e^{\frac{1}{2}-\gamma}}{\kappa v_{12}\left|\sin \frac{1}{2} \Omega\left(t-t_{0}\right)\right|}\right],
$$

giving recurrences, and no secular evolution for $\mathscr{E}^{V}$ :

$$
\mathscr{E}^{r} \approx 0 \quad \text { (secular part). }
$$

Similarly, if we are considering times short compared to the characteristic (hybrid) time scale of evolution of the velocity distribution $\varphi$, the use of $J$ is appropriate. Or, if for one of a number of possible reasons (cf. $\$ 3.3$ above) a Markovian approximation is reasonable, (4.6) again would be a valid result.

In particular, if

$$
|\Omega|\left(t-t_{0}\right) \ll 1 \text { and } \kappa v_{12}\left(t-t_{0}\right) \ll 1 \text {, }
$$

then

$$
\operatorname{Tr} \mathbf{J} \approx\left(t-t_{0}\right)^{2} \ln \left[\frac{2 e^{\frac{1}{2}-\gamma}}{\kappa v_{12}\left(t-t_{0}\right)}\right] .
$$

On the other hand, in the case

$$
a=\kappa v_{12} /|\Omega| \gg 1,
$$

the $K_{1}$ Bessel function is exponentially small except for the expected recurrences: $\operatorname{Tr} \mathbf{J} \approx \frac{2}{\kappa^{2} v_{12}^{2}}\left\{1-\left[\left(\frac{\pi}{2}\right)^{\frac{t}{2}}\left(\left|\frac{2 \kappa v_{12}}{\Omega} \sin \frac{1}{2} \Omega\left(t-t_{0}\right)\right|\right)^{\frac{1}{2}} \exp \left(-\left|\frac{2 \kappa v_{12}}{\Omega} \sin \frac{1}{2} \Omega\left(t-t_{0}\right)\right|\right)\right]\right\}$. 


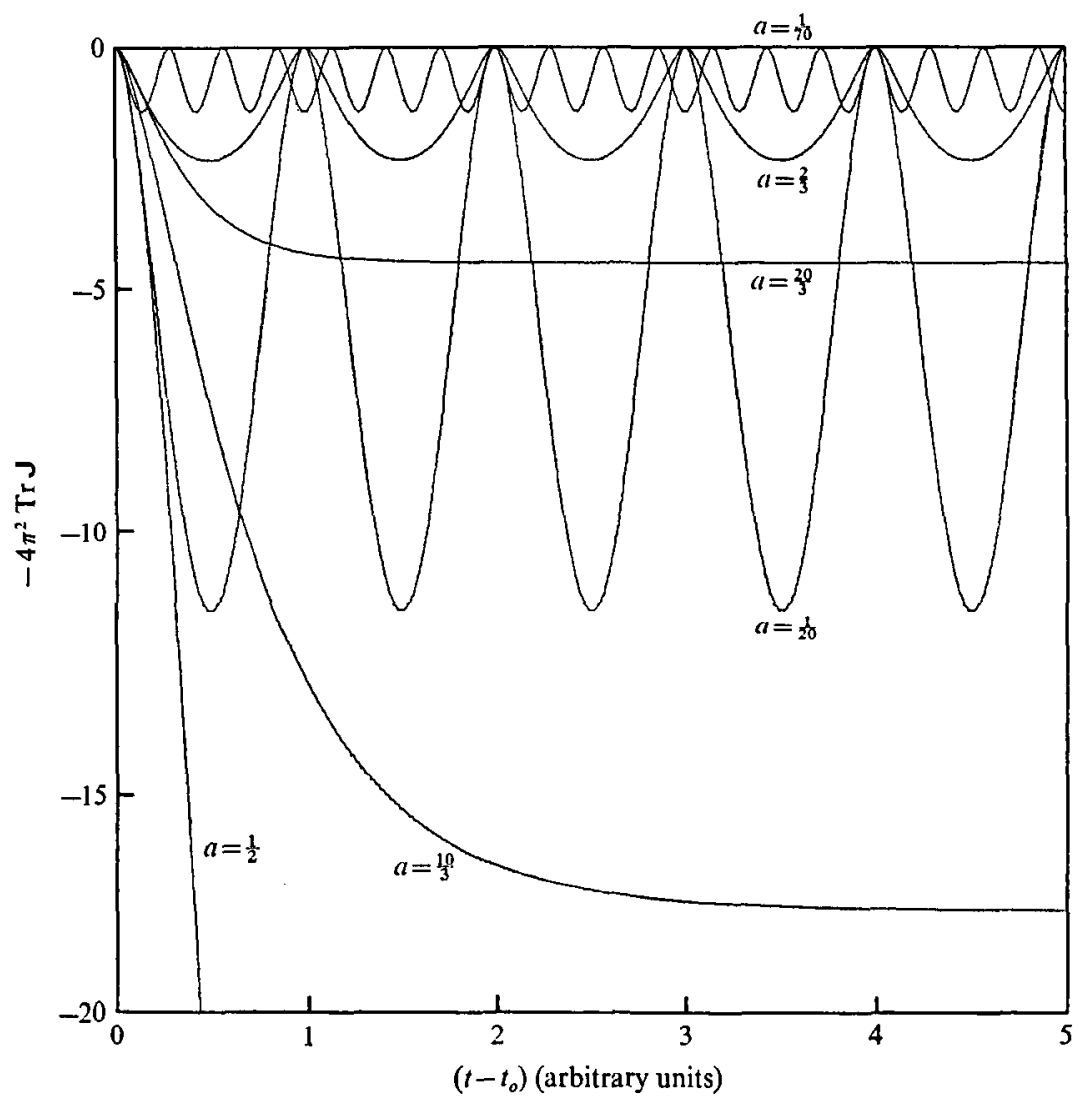

Figure 1. A plot of $[-\operatorname{Tr}]]$ as a function of time.

Neglecting the recurrences, one sees a fast relaxation to a constant value

corresponding to $(3.34)$ :

$$
\operatorname{Tr} J \approx 2 / \kappa^{2} v_{13}^{2},
$$

$$
\mathscr{E}^{\nabla} \approx-\left[2 \pi C / \kappa^{2}\right] \int d \mathbf{v}[\varphi(v)]^{2},
$$

and

$$
d \mathscr{E} \mathrm{V} / d t=0=-d \mathscr{E}^{\mathrm{K}} / d t
$$

to first order in the coupling parameter.

The interest of the more general form (4.4) for $\operatorname{Tr} J$, containing the dominant time-dependence of $\mathscr{E}^{V}$ (according to (4.6)), is that it is rather easy to match the various ranges and to get a consistent picture without taking limits. Figure 1 gives a plot of [ $-\mathrm{Tr} \mathrm{J}]$, the main kernel of the integral for the correlational energy density (4.6), when there are no initial correlations: $g\left(t_{0}\right)=0$. The ordinate is scaled up by an arbitrary factor $4 \pi^{2}$.

In identifying the diagram as a schematic plot of $\mathscr{E}^{V}(t)$, or $\mathscr{E}^{\mathrm{K}}\left(t_{0}\right)-\mathscr{E}^{\mathrm{K}}(t)$ (a moment of the velocity distribution), it should be realized that 'quasi-stroboscopic' corrections (\$4.4) are not included.

The three graphs with the same steep slopes near the origin correspond to 
$2 \pi / \kappa v_{12}=20$, the two with gradual slopes, to $2 \pi / \kappa v_{12}=\frac{3}{2}$, and the one with an intermediate slope, to $2 \pi / \kappa v_{12}=3$. One graph has a short period $2 \pi /|\Omega|=\frac{2}{7}$, two have $2 \pi /|\Omega|=1$, and the others contain half a period: $2 \pi /|\Omega|=10$. The corresponding values of $a=[2 \pi /|\Omega|] /\left[2 \pi / \kappa v_{12}\right]$ are indicated.

There is an interesting continuous transition from oscillations to relaxation that does not involve damped oscillations; we suggest it as a simplified model of a partial transition from reversible to irreversible behaviour.

\subsection{Discussion of irreversibility}

Has 'true irreversibility' been found without the introduction of an asymptotic time limit? The question seems to require more precision. The existence of a long-lived dependence of a moment $\mathscr{E}^{\mathrm{K}}$ of $\varphi(v)$ on the initial time $t_{0}$ is a distinctive feature not present in Markovian versions with cut-offs $T_{0}$ (or $\left[\kappa v_{12}\right]^{-1}$, etc.) The mirror diagram to figure 1 in the region $t<t_{0}$ would give natural continuations to all the graphs. This situation, suggesting reversibility, is in contrast to some otherwise similar gravitational cases, where the removal of short-lived initial transients would give non-zero slopes at the origin. In the latter cases, the branch of the time-reversed evolution (as measured by $\mathscr{E}^{V}=\mathscr{E}^{\mathrm{K}}\left(t_{0}\right)-\mathscr{E}^{\mathrm{K}}[\varphi]$ ) is more clearly distinct from that of the normal evolution.

In the present case, it can be said that the system has the character of irreversibility in the following sense. In the limit $\Omega \rightarrow 0$, for small $\kappa$,

$$
\mathscr{E}^{\mathrm{K}}\left(t_{2}\right)>\mathscr{E}^{\mathrm{K}}\left(t_{1}\right) \text { for }\left|t_{2}-t_{0}\right|>\left|t_{1}-t_{0}\right|,
$$

and when also $\kappa \rightarrow 0, \exp \left\{-\mathscr{E}^{\mathrm{K}}[\varphi]\right.$ /const. $\}$ is an appropriate Liapunov function measuring the approach to an infinitely dispersed velocity distribution. But though there is no symmetry-breaking by external perturbations, it should be realized that such a relaxation might be argued to be just a slight generalization of one of the usual phase-mixing transients; the equations retain echoes at $t=t_{0}+2 \pi /|\Omega|$ even if $2 \pi /|\Omega|$ is much larger than any other characteristic time (or any experimental times).

Let us therefore be cautious. Nevertheless we assert that the introduction of a special initial condition such as $g\left(t_{0}\right)=0$ is a key ingredient for apparentlydissipative behaviour to be observable. The 'mathematical trickery' of eliminating odd functions by limiting procedures such as

$$
\left(k v_{12}-\omega\right)^{-1} \rightarrow\left(k v_{12}-i \epsilon\right)^{-1} \rightarrow \pi \delta\left(k v_{12}\right)
$$

would seem to be less important. The consideration of such limits might make irreversible behaviour more explicit, and the transitions between qualitatively different behaviours sharper. But the limits themselves do not 'cause' the timereversal symmetry to be broken. Similar conclusions have been reached by various authors in the study of both Landau damping (a mixing of phases) and 'dynamical irreversibility.'

Let us also note that the elimination of recurrences by taking $\Omega \rightarrow 0$ is qualitatively more 'drastic' than the corresponding elimination of Poincaré recurrences by taking $N \rightarrow \infty$, because in our present approximations the ensemble 
as a whole, as measured by $\mathscr{E}^{\mathrm{K}}[\varphi]$, evolves periodically, with a discrete frequency $\Omega \neq 0$; the individual systems composing the ensemble have a common period. Still, each special case, no matter how degenerate sheds light on a question that has proved surprisingly difficult over the decades. Part of the problem has been to justify the convenient choice of the order of limiting procedures; an advantage of the present models is that fewer limits than usual need be taken to clarify the final results. As a guide to how somewhat similar models have been situated within the point of view of the Brussels school of non-equilibrium statistical mechanics, we refer to Rae \& Davidson (1973), Rae (1973), Moffat (1974) and Guo \& Guo (1975).

The focus of attention on the moment $\mathscr{E}^{\mathrm{K}}$ of $\varphi$, normally conserved in weakcoupling approximations, must in the context of a discussion of irreversibility be considered a simplification. For a brief discussion of the non-periodic evolution (even for large $\Omega$ ) of other moments of the velocity distribution in the stroboscopic approximation, and the status of the $H$-theorem, see $\S 3.2(b)$ above. Vahala \& Montgomery (1971) pointed out that the $H$-theorem is strictly valid for their version of the kinetic equation.

One cannot, of course, dismiss the initial condition $g\left(t_{0}\right)=0$ as being too unnatural to apply to real freely-evolving systems. It is a reasonable point of view that the introduction of a screened potential (with a parameter $\kappa \neq 0$ ) models an initial correlation. One is free to construct an ensemble of computersimulation experiments with no further initial correlations.

\subsection{Quasi-stroboscopic approximation}

As promised, we now return to the general expression (4.2) for the correlational energy density $\mathscr{E} V$, and go one step beyond the stroboscopic approximation (3.7), (3.8), in order to improve the negative result (4.9) by extracting a significant secular evolution over several whole gyroperiods. We take into account the mean evolution of $\varphi\left(\left|\mathbf{v}_{1}\right|\right) \varphi\left(\left|\mathbf{v}_{2}\right|\right)$ during a gyroperiod, but not its oscillation:

$$
\begin{aligned}
& \varphi \varphi\left(t-\pi|\Omega|^{-1}[2 \mu-1]-\delta \tau\right) \approx \varphi \varphi\left(t-\pi|\Omega|^{-1}[2 \mu-1]\right) \\
& \quad-\delta \tau(\partial / \partial t) \varphi \varphi\left(t-\pi|\Omega|^{-1}[2 \mu-1]\right), \quad|\delta \tau| \leqslant \pi|\Omega|^{-1}, \quad \mu=1,2,3, \ldots
\end{aligned}
$$

After a calculation similar to the previous ones (some additional intermediate steps of these calculations appear in preliminary notes available from the author), we find that

$$
\mathscr{E}^{V}=\frac{2 C}{\Omega^{2}} \int d \mathbf{v}_{1} d \mathbf{v}_{2}\left[I_{0}(a) K_{0}(a)-I_{1}(a) K_{1}(a)\right]\left\{\varphi\left(v_{1}, t\right) \varphi\left(v_{2}, t\right)-\varphi\left(v_{1}, t_{0}\right) \varphi\left(v_{2}, t_{0}\right)\right\}
$$

In the limit of small $\kappa$, the expression in the square brackets involves a factor $\ln [1 / \kappa]$, but that fails to contribute because

$$
\int d \mathbf{v} \varphi(v, t)=1=\int d \mathbf{v} \varphi\left(v, t_{0}\right)
$$

We get

$$
\mathscr{E}_{\kappa \rightarrow 0}=\frac{-8 \pi q^{4} n_{0}^{2}}{m l^{3} \Omega^{2}} \int d \mathbf{v}_{1} d \mathbf{v}_{2}\left[\ln \frac{u(t)}{v_{12}}\right] \varphi\left(v_{1}, t_{0}\right) \varphi\left(v_{2}, t_{0}\right)
$$


where $u(t)$ is defined by

$$
\int d \mathbf{v}_{1} d \mathbf{v}_{2}\left[\ln \frac{u(t)}{v_{12}}\right] \varphi \varphi(t)=0 .
$$

Thus $\mathscr{E}_{k \rightarrow 0}^{V}$ stops changing when the logarithmic moment of $\varphi \varphi$ has relaxed. Its asymptotic value, including the sign, depends primarily on the shape of the initial velocity distribution.

It will be interesting to see if future computer simulations find a range of parameters where the indicated memory of the initial velocity distribution remains unaltered by collective or boundary effects.

The predicted effect becomes small when $\Omega$ is so large that not much work can be done by the Coulomb force. It gives, however, an estimate of the error in using the kinetic-energy-conserving kinetic equation (3.10), (3.21), and moreover is proportional to some related results to be found in the next section.

It is to be mentioned that the prefix 'quasi' in quasi-stroboscopic approximation, an expression coined here, is derived from a superficial resemblance of some related formulae to those pertaining to non-resonant wave-particle interactions in quasi-linear theory (e.g. Sagdeev \& Galeev 1969). But I am not prepared to argue that the resemblance has any deep significance, or that the approximation would turn out to be as important as the quasi-linear approximation.

Some information on the dominant range-distribution of the potential energy is suggested by the form of the result (4.20). The functions $K_{0}$ and $K_{1}$ contain factors that are exponentially small for large $a$. Thus as $\kappa^{-1}$ is reduced to the scale of typical inverse gyroradii, the effect begins to be cut off; but for larger $\kappa^{-1}$ there is only a weak direct dependence on $\kappa$. We take it that the correlational energy spectrum involves relatively short ranges, not essentially related to the longrange cut-off. (See (3.25), etc. for a similar argument; see \$4.2. for qualitatively different contributions to $\mathscr{E} V$.) Some interesting quantities found in the next section will be proportional to the magnitude of this short-range effect, but will apparently have larger characteristic length scales.

\section{Other moments of the correlation function}

\subsection{Primary motivation}

We consider additional moments that share with $\mathscr{E}^{V}$ the property of having no weights with respect to directions perpendicular to B. Our choice of moments is mainly governed by the desire to understand the microstructure that other approaches suggest might spontaneously appear. In particular, in the limit $\Omega \rightarrow \infty$, the guiding-centre velocities are constrained, and there can be a 'negative temperature instability' generating vortices ( $\$ 1.2$ above). We shall see that some generation of microvorticity is predicted already by our homogeneous, isotropic model with finite $\Omega$ and no collective effects. 


\subsection{A second average of relative positions}

The calculation of

$$
\mathscr{E}^{V}=\frac{1}{2} \rho N\left\langle V^{12}\right\rangle_{\text {corr }}
$$

defined by (4.1), gave a limited amount of information concerning the rangedependence of the correlations. To gain further insight, and to derive some results that can be used for consistency checks, we now calculate the similarly defined quantity

$$
\left\langle\mathbf{x}_{12} \mathbf{F}_{12}\right\rangle_{\text {corr }}=\frac{L^{2}}{N^{2}} \int d \mathbf{v}_{1} d \mathbf{v}_{2} \int d \mathbf{x}_{12} \mathbf{x}_{12} \mathbf{F}_{12}\left(\mathbf{x}_{12}\right) g(1,2),
$$

where

$$
\mathbf{F}_{12}\left(\mathbf{x}_{12}\right)=-\nabla V^{12}
$$

as in (2.2). It will develop that $-\left\langle\mathbf{x}_{12} \nabla V^{12}\right\rangle_{\text {corr }}$ does not provide much additional spectral information on $\left\langle V^{12}\right\rangle_{\text {corr }}$ when $\kappa$ is small; nevertheless the results of this subsection will provide a useful prelude to what follows.

By taking Fourier transforms and differentiating with respect to parameters, one can again do the spatial integration, and obtain

$$
\begin{aligned}
\rho N\left\langle\mathbf{x}_{12} \mathbf{F}_{12}\right\rangle_{\text {corr }}=\frac{1}{2} C & \int d \mathbf{v}_{1} d \mathbf{v}_{2} \int_{0}^{t-t_{0}} d \tau \frac{1}{\kappa}\left[K_{0}\left(Y_{\tau}\right)\left(\hat{x} \mathbf{Y}_{\tau} \hat{x}+\hat{y} \mathbf{Y}_{\tau} \hat{y}+\mathbf{Y}_{\tau} \mathbf{I}-\mathbf{I} \mathbf{Y}_{\tau}\right)\right. \\
& \left.-K_{1}\left(Y_{\tau}\right) \frac{\mathbf{Y}_{\tau} \mathbf{Y}_{\tau} \mathbf{Y}_{\tau}}{Y_{\tau}}\right] \cdot \mathbf{R}(\tau) \cdot \partial_{12} \varphi\left(v_{1}, t-\tau\right) \varphi\left(v_{2}, t-\tau\right)
\end{aligned}
$$

Since the off-diagonal elements of (5.2) clearly vanish, let us restrict attention to $\left\langle x_{12 x} F_{12 x}\right\rangle_{\text {corr }}$. In the stroboscopic approximation (3.7), the secular part is

$$
\rho N\left\langle x_{12 x} F_{12 x}\right\rangle_{\text {corr }}=\frac{1}{2} C \int d \mathbf{v}_{1} d \mathbf{v}_{2} \mathbf{W} \cdot \partial_{12}\left\langle\varphi\left(v_{1}\right) \varphi\left(v_{2}\right)\right\rangle_{t},
$$

where $\mathbf{W}$, implicitly defined by (5.4), works out to be

$\mathrm{W}=-\frac{2\left(t-t_{0}\right)}{\Omega} \frac{v_{12 y}^{2}}{v_{12}^{2}}\left\{-I_{1}(a) K_{1}(a)+a I_{0}(a) K_{1}(a)-a I_{1}(a) K_{0}(a)\right\}\left(v_{12 x} \hat{y}-v_{12 y} \hat{x}\right)$.

Because of the odd parity in $v_{12},(5.5)$ reduces to

$$
\rho N\left\langle x_{12 x} F_{12 x}\right\rangle_{\text {corr }}=0 \quad \text { (stroboscopic, secular part). }
$$

In the quasi-stroboscopic approximation (4.19), we have

$$
\rho N\left\langle x_{12 x} F_{12 x}\right\rangle_{\text {corr }}=C \int d \mathbf{v}_{1} d \mathbf{v}_{2}\left[\varphi \varphi(t)-\varphi \varphi\left(t_{0}\right)\right] \frac{\partial}{\partial \mathbf{v}_{12}} \cdot \tilde{W},
$$

with the $\tau$-integral contained in the definition of $\mathbf{W}$ again tractable with some effort. It yields

$$
\begin{aligned}
\tilde{W}= & \frac{2}{\Omega^{2}} \frac{v_{12 x}^{2} v_{12}}{v_{12}^{2}}\left\{I_{0}(a) K_{0}(a)-I_{1}(a) K_{1}(a)-\frac{2}{a} I_{1}(a) K_{0}(a)\right\} \\
& -\frac{2}{\Omega^{2}}\left(-v_{12 x} \hat{x}+v_{12 y} \hat{y}\right)\left\{I_{0}(a) K_{0}(a)-I_{1}(a) K_{1}(a)-\frac{2}{a} I_{1}(a) K_{0}(a)\right. \\
& \left.+\frac{1}{2 a^{2}}-\frac{1}{a^{2}} I_{1}(a) K_{1}(a)\right\} .
\end{aligned}
$$


Taking the divergence indicated in (5.8), and using the identity

$$
\begin{aligned}
\int d \mathbf{v}_{1} d \mathbf{v}_{2}\left(\lambda v_{12 x}^{2}\right. & \left.+\mu v_{12 y}^{2}+\xi v_{12 x}^{4}+\eta v_{12 x}^{2} v_{12 y}^{2}+\zeta v_{12 y}^{4}\right) \alpha\left(v_{1}, v_{2}, v_{12}\right) \\
= & \int d \mathbf{v}_{1} d \mathbf{v}_{2}\left[\left(\frac{1}{2} \lambda+\frac{1}{2} \mu\right) v_{12}^{2}+\left(\frac{3}{8} \xi+\frac{1}{8} \eta+\frac{3}{8} \zeta\right) v_{12}^{4}\right] \alpha\left(v_{1}, v_{2}, v_{12}\right)
\end{aligned}
$$

we find

$$
\frac{\partial}{\partial \mathbf{v}_{12}} \cdot \tilde{W}=\frac{1}{2} \partial_{12} \cdot \tilde{W}=-\frac{1}{\Omega^{2}} M^{(2)}(a)+X^{(2)}
$$

where

$$
M^{(2)}(a)=2\left[a I_{0}(a) K_{1}(a)-a I_{1}(a) K_{0}(a)-I_{1}(a) K_{1}(a)\right]
$$

and $X^{(2)}$ is a quantity that fails to contribute to (5.8) because of $(5.10)$ :

$$
\rho N\left\langle x_{12 x} F_{12 x}\right\rangle_{\text {corr }}=-\frac{C}{\Omega^{2}} \int d v_{1} d \mathbf{v}_{2} M^{(2)}\left(\kappa v_{12} /|\Omega|\right)\left[\varphi \varphi(t)-\varphi \varphi\left(t_{0}\right)\right] .
$$

In the limit $\kappa \rightarrow 0, M^{(2)}(a) \rightarrow 1$, and we have immediately

$$
\rho N\left\langle x_{12 x} F_{12 x}\right\rangle_{\text {corr }}=0 \quad(\kappa \rightarrow 0, \text { quasi-stroboscopic }) .
$$

As distinct from (5.7), which is typical, (5.14) gives a helpful consistency check. For in the limit $\kappa \rightarrow 0$ of Coulomb forces between the rods, we have simply

$$
\mathbf{x}_{12} \mathbf{F}_{12}=\left(2 q^{2} / m l\right) \widehat{x}_{12} \widehat{x}_{12}
$$

Thus

$$
\left\langle\mathbf{x}_{12} \mathbf{F}_{12}\right\rangle_{\mathrm{corr}}=\frac{\int d \mathbf{v}_{1} d \mathbf{v}_{2} d \mathbf{x}_{1} d \mathbf{x}_{2}\left(2 q^{2} / m l\right) \hat{x}_{12} \hat{x}_{12} g(1,2)}{\int d \mathbf{v}_{1} d \mathbf{v}_{2} d \mathbf{x}_{1} d \mathbf{x}_{2} f(1) f(2)} .
$$

In (5.16), the off-diagonal elements are zero, and the diagonal elements are both equal to half the trace. Since $\hat{x}_{12} \cdot \hat{x}_{12}=1,(5.16)$ vanishes identically. Equation (5.14) shows the preservation of this property by the quasi-stroboscopic approximation.

The asymptotic behaviour for small $\kappa$ contains a coefficient $\kappa^{2}$, in the quasistroboscopic approximation, and can be related to $\mathscr{E}^{\mathrm{V}}$ by pleasingly simple formulae:

$$
\begin{aligned}
& \rho N\left\langle x_{12 x} F_{12 x}\right\rangle_{\text {corr }} \approx-\frac{5 C \kappa^{2}}{2 \Omega^{4}} \int d \mathbf{v}_{1} d \mathbf{v}_{2}\left[\varphi \varphi(t)-\varphi \varphi\left(t_{0}\right)\right] \\
& \times v_{12}^{2}\left\{\ln \left[\left(2|\Omega| / \kappa v_{12}\right) \exp (-\gamma+1 / 20)\right]\right\} \quad(\kappa \text { small, } \gamma=0.5772) \\
& \frac{\left\langle-\mathbf{x}_{12} \cdot \nabla V^{12}\right\rangle_{\text {corr }}}{\left\langle V^{12}\right\rangle_{\text {corr }}} \approx\left[\frac{5 C \kappa^{2}}{2 \Omega^{4} \mathscr{E} V} \int d v_{1} d \mathbf{v}_{2} v_{12}^{2}\left\{\ln \frac{\tilde{u}(t)}{v_{12}}\right\} \varphi \varphi\left(t_{0}\right)\right] \\
&+\left[\frac{10 C \kappa^{2}}{\rho \Omega^{4}} \ln \frac{2|\Omega| \exp (-\gamma+1 / 20)}{\kappa \tilde{u}(t)}\right]
\end{aligned}
$$

where $\tilde{u}(t)$ is defined in analogy to $u(t)$ in $(4.23)$ :

$$
\int d \mathbf{v}_{1} d \mathbf{v}_{2} v_{12}^{2}\left[\ln \frac{\tilde{u}(t)}{v_{12}}\right] \varphi \varphi(t)=0 .
$$


Substituting (4.22) for $\mathscr{E}^{V}$ on the right side of (5.18) gives the form

$$
\begin{aligned}
\frac{\rho N\left\langle x_{12 x} F_{12 x}\right\rangle_{\text {corr }}}{\mathscr{E}^{V}} \approx & \frac{-5 \kappa^{2} \int d \mathbf{v}_{1} d \mathbf{v}_{2} v_{12}^{2}\left[\ln \tilde{u}(t) / v_{12}\right] \varphi \varphi\left(t_{0}\right)}{4 \Omega^{2} \int d \mathbf{v}_{1} d \mathbf{v}_{2}\left[\ln u(t) / v_{12}\right] \varphi \varphi\left(t_{0}\right)} \\
& +\frac{10 C^{\prime} \kappa^{2}}{\Omega^{4}} \ln \frac{2|\Omega| \exp (-\gamma+1 / 20)}{\kappa \tilde{u}(t)}
\end{aligned}
$$

(quasi-stroboscopic, $\kappa$ small).

Again, the primary dependence is on $\varphi\left(t_{0}\right)$ rather than $\varphi(t)$, providing that $u(t)$ and $\tilde{u}(t)$ vary sufficiently slowly for large times. To the extent that the $H$-theorem is approximately valid for our version of the theory (cf. $\$ \S 3.2(b)$ and 4.3 ), (5.20) predicts a relaxation to a time-independent value for the ratio of the two spatial averages, amenable to testing.

\subsection{Averages with velocity-dependent weighting factors}

We now examine whether two rods tend to revolve around each other as they interact. We shall find some indications that the system develops vortices, even when it is statistically homogeneous and isotropic (and therefore $\nabla \times\langle v\rangle=0$, $(\nabla n) \times\langle v\rangle=0$ ). Let us sometimes also use the word microvorticity, to suggest an incoherence as to the location of the vortices from one member of the statistical ensemble to another, in the infinite system with a cut-off potential. But if $\kappa \approx L^{-1}$, then the long ranges suggested by some of the results would imply macroscopic characteristic length scales.

It will not be surprising that the average kinetic energy of pairs with large potential energy is found to deviate from that of separate pairs, as correlations develop. Some of the formulae are in fact quite simple (cf. (5.34), (5.15)):

$$
\begin{gathered}
\left\langle\mathbf{v}_{12} \mathbf{v}_{12}\right\rangle_{\text {corr }}=0 \quad \text { (stroboscopic, secular part), } \\
\left.\frac{N n_{0} m}{4 l}\left\langle v_{12}^{2}\right\rangle_{\text {corr }}=\frac{2 \omega_{\mathrm{p}}^{2}}{\Omega^{2}} \mathscr{E} V(t) \quad \text { (quasi-stroboscopic, } \kappa \rightarrow 0\right),
\end{gathered}
$$

where

$$
\omega_{\mathrm{p}}^{2}=4 \pi \frac{n_{0} m}{l} \frac{q^{2}}{m^{2}}=\left(4 \pi n_{0} C^{\prime}\right)^{\frac{1}{2}}
$$

But the relative-velocity anisotropy with respect to the direction of separation would appear to be more interesting, especially in the light of the computer simulations of Joyce \& Montgomery (1973), in the guiding-centre approximation. Such vorticity could develop into macroscopic motion associated with rapid transport. 
(a) Stroboscopic approximation, secular part

In the stroboscopic approximation, many null results for the secular parts of such averages may be obtained, because of the many symmetries of the model. For instance,

$$
\begin{aligned}
&\left\langle\mathbf{v}_{12} F_{12}\right\rangle_{\text {corr }}=0, \\
&\left\langle v_{12} \mathbf{v}_{12} \mathbf{v}_{12} F_{12}\right\rangle_{\text {corr }}=0, \\
&\left\langle v_{12} \mathbf{v}_{12} V^{12}\left(x_{12}\right)\right\rangle_{\text {corr }}=0, \\
&\left\langle v_{12} \mathbf{v}_{12} \mathbf{x}_{12} F_{12}\right\rangle_{\text {corr }}=0 .
\end{aligned}
$$

On the other hand, if the averages contain direction-dependent weights, as in (2.7), they will lead to non-zero results such as the right-hand side of the kinetic equation (3.10).

(b) Quasi-stroboscopic approximation

Let us focus attention on the quantity $\left\langle\mathbf{v}_{12} \mathbf{v}_{12} \mathbf{x}_{12} \mathbf{F}_{12}\right\rangle_{\text {corr }}$, and study the correction to (5.27). In analogy to (5.8) above, we have

$$
\rho N\left\langle v_{12 y}^{2} x_{12 x} F_{12 x}\right\rangle_{\text {corr }}=C \int d \mathbf{v}_{1} d \mathbf{v}_{2}\left[\varphi \varphi(t)-\varphi \varphi\left(t_{0}\right)\right] \frac{\partial}{\partial \mathbf{v}_{12}} \cdot v_{12 y}^{2} \mathbf{W} .
$$

Moreover,

$$
\frac{\partial}{\partial \mathrm{v}_{12}} \cdot v_{12 \nu}^{2} \mathrm{~W}=\frac{-v_{12}^{2}}{2 \Omega^{2}}\left\{K_{0}(a)\left[\frac{2}{a}\left(2-a^{2}\right) I_{1}(a)-I_{0}(a)\right]-1\right\}+X^{(3)},
$$

where $X^{(3)}$ is a part that fails to contribute to (5.28), because of (5.10).

In the limit of small $\kappa$,

Thus

$$
\frac{\partial}{\partial \mathrm{v}_{12}} \cdot v_{12 y}^{2} \mathbf{W}=\frac{-v_{12}^{2}}{2 \Omega^{2}}\left[\ln \frac{2 e^{-\gamma-1}|\Omega|}{\kappa v_{12}}\right]+O\left(a^{2} \log \frac{1}{a}\right)+X_{\kappa \rightarrow 0}^{(3)} .
$$

$$
\begin{aligned}
\rho N\left\langle v_{12 \nu}^{2} x_{12 x} F_{12 x}\right\rangle_{\text {corr }} & \\
= & -\frac{C}{2 \Omega^{2}}\left[\ln \frac{2 e^{-\gamma-1}|\Omega|}{\kappa \tilde{u}(t)}\right] \int d \mathbf{v}_{1} d v_{2} v_{12}^{2}\left[\varphi \varphi(t)-\varphi \varphi\left(t_{0}\right)\right] \\
& +\frac{C}{2 \Omega^{2}} \int d \mathbf{v}_{1} d \mathbf{v}_{2} v_{12}^{2}\left[\ln \frac{\tilde{u}(t)}{v_{12}}\right] \varphi \varphi\left(t_{0}\right), \quad(\kappa \text { very small }) .
\end{aligned}
$$

Similarly,

$$
\rho N\left\langle v_{12}^{2} x_{12 x} F_{12 x}\right\rangle_{\mathrm{corr}}=-\frac{2 C}{\Omega^{2}} \int d \mathbf{v}_{1} d \mathbf{v}_{2} v_{12}^{2}\left[\varphi \varphi(t)-\varphi \varphi\left(t_{0}\right)\right] M^{(4)}(a),
$$

where

$$
M^{(4)}(a)=1+K_{0}(a)\left[\frac{2}{a}\left(1-a^{2}\right) I_{1}(a)-I_{0}(a)\right] .
$$

The $\kappa \rightarrow 0$ limit has no logarithmic part:

$$
\rho N\left\langle v_{12}^{2} x_{12 x} F_{12 x}\right\rangle_{\mathrm{corr}}=-\frac{2 C}{\Omega^{2}} \int d \mathbf{v}_{1} d \mathbf{v}_{2} v_{12}^{2}\left[\varphi \varphi(t)-\varphi \varphi\left(t_{0}\right)\right] \quad(\kappa \rightarrow 0) .
$$


Subtracting, we find a correlation between the direction of separation of pairs and the direction of the relative velocity between them. The correlation increases logarithmically with $\kappa^{-\mathbf{1}}$ :

$$
\begin{aligned}
& \rho N\left\langle\left(v_{12 y}^{2}-\frac{1}{2} v_{12}^{2}\right) x_{12 x} F_{12 x}\right\rangle_{\mathrm{corr}} \\
&=\left(C / 2 \Omega^{2}\right) \int d \mathbf{v}_{1} d \mathbf{v}_{2} v_{12}^{2}\left\{3-K_{0}(a)\left[I_{0}(a)+2 a I_{1}(a)\right]\right\}\left[\varphi \varphi(t)-\varphi \varphi\left(t_{0}\right)\right], \\
& \rho N\left\langle\left(v_{12 y}^{2}-\frac{1}{2} v_{12}^{2}\right) x_{12 x} F_{12 x}\right\rangle_{\mathrm{corr}} \\
&=\left\{-\frac{C}{2 \Omega^{2}}\left[\ln \frac{2 e^{-\gamma-3}|\Omega|}{\kappa \tilde{u}(t)}\right] \int d \mathbf{v}_{1} d \mathbf{v}_{2} v_{12}^{2}\left[\varphi \varphi(t)-\varphi \varphi\left(t_{0}\right)\right]\right\} \\
& \quad+\left\{\frac{C}{2 \Omega^{2}} \int d \mathbf{v}_{1} d \mathbf{v}_{2} v_{12}^{2}\left[\ln \frac{\tilde{u}(t)}{v_{12}}\right] \varphi \varphi\left(t_{0}\right)\right\}, \quad(\kappa \text { very small }) .
\end{aligned}
$$

The quantities $C$ and $\tilde{u}(t)$ were defined by (3.5) and (5.19). One should also have in mind the auxiliary expressions

$$
-\frac{1}{4} \rho \int d \mathbf{v}_{1} d \mathbf{v}_{2} v_{12}^{2}\left[\varphi \varphi(t)-\varphi \varphi\left(t_{0}\right)\right]=\mathscr{E}^{K}\left(t_{0}\right)-\mathscr{E}^{K}(t)=\mathscr{E}^{V}(t)=\frac{1}{2} \rho N\left\langle V^{12}\left(x_{12}\right)\right\rangle_{\mathrm{corr}}
$$

In (5.36), there is a coefficient $\Omega^{-2}$, making the predicted effect decrease with smaller gyroperiod/hybrid-time-scale ratios.

But the most salient feature is the existence of a term $\propto \ln 1 / \kappa$ (without a coefficient $\kappa^{2}$ as in (5.20)). It is reasonable to suggest that the weak-coupling approximation, with statistical homogeneity and isotropy, already yields a development of long-range vorticity, much the same as has been found with computer simulations in the limit $\Omega \rightarrow \infty$ with special boundaries. Equations (5.36) and (5.37) indicate that the amplitude should be linked to the amplitude of the short-range correlational energy (4.22). It is to be remembered that such energy could have a sign such that the mutually repulsive electric charges would be clustered.

We should however note that such $\kappa$-divergent results can be generated in abundance just by increasing the weight for large separations. For instance $\left\langle\mathbf{v}_{12} \mathbf{x}_{12}\right\rangle_{\text {corr }}$ seems too sensitive to the boundary conditions and to the order of the limits to be meaningfully calculated. On the other hand, indications of microvorticity, without factors $\ln [1 / \kappa]$, appear in other choices for measurable quantities. For example,

$$
\left\langle\sin ^{2}\left(\arg \hat{v}_{12}-\arg \hat{x}_{12}\right)\right\rangle_{\text {corr }} \quad(\kappa \rightarrow 0)=\frac{\omega_{\mathrm{p}}^{2}}{2 N \Omega^{2}} \int d \mathbf{v}_{1} d \mathbf{v}_{2}\left[\ln \frac{u(t)}{v_{12}}\right] \varphi \varphi\left(t_{0}\right),
$$

in proportion to $\mathscr{E}^{V}(t)$ (cf. (4.22)). Also,

$$
\begin{aligned}
N \rho\left\langle\mathrm{F}_{12} \mathbf{v}_{12}\right\rangle_{\text {corr }} & =\frac{2 C}{\Omega}\left(\begin{array}{rr}
0 & 1 \\
-1 & 0
\end{array}\right) \int d \mathbf{v}_{1} d \mathbf{v}_{2}\left[\varphi \varphi(t)-\varphi \varphi\left(t_{0}\right)\right] \\
\times & {\left[I_{0}(a) K_{0}(a)-2 I_{1}(a) K_{1}(a)-a I_{1}(a) K_{0}(a)+a I_{0}(a) K_{1}(a)\right] }
\end{aligned}
$$

and so

$$
N \rho\left\langle\mathbf{F}_{12} \mathbf{v}_{12}\right\rangle_{\text {corr }}(\kappa \rightarrow 0)=\Omega\left(\begin{array}{rr}
0 & 1 \\
-1 & 0
\end{array}\right) \mathscr{E}^{\mathscr{V}}(t)
$$

or

$$
\left\langle v_{12 y} \frac{x_{12 x}}{x_{12}^{2}}\right\rangle_{\text {corr }}(\kappa \rightarrow 0)=\frac{-\omega_{\mathrm{p}}^{2}}{\Omega N} \int d \mathbf{v}_{1} d \mathbf{v}_{2}\left[\ln \frac{u(t)}{v_{12}}\right] \varphi \varphi\left(t_{0}\right) \text {. }
$$


It would be interesting to test cross-relations such as (5.40) (and (5.22)), independently of the detailed dependence on $\varphi\left(t_{0}\right)$ given by (5.41), (4.22). Perhaps there is an effective-initial-time function $t_{0}^{\text {eff }}(t)$ that would not appear explicitly in the cross-relations, but would preserve them even as memory of past conditions is forgotten.

\section{Further discussion}

\subsection{Time scales and tests by computer simulation}

The present model predicts a continued sensitivity to the time elapsed since conditions were as initially prescribed. If simulations show otherwise, it might be possible to isolate the effects not taken into account here. It is not at present clear that there should be a cut-off $T_{0}$ associated with the diffusion of a certain pair beyond a region of 'resonance'; pairs entering 'resonance' should also be considered. The correlational energy density, and related quantities discussed in $\S 5$, seem particularly suitable for consideration in tests of memory retention.

The non-logarithmic dependences on the length and time scales are especially important to test. It does not appear promising to examine the parameters inside logarithm factors by simulation; fine features of the results might be unstable to external symmetry-breaking perturbations. But the dominant $B$-dependence should be testable, and particularly sensitive to the details of the model and the approximations made. We would expect the most favourable comparisons for the ranges where the time scales are not well separated, but the magnetic field is weak enough so that quasi-stroboscopic effects could be as important as strong-coupling corrections. We have been careful to keep $\kappa\left\langle v_{12}\right\rangle / \Omega$ and $\omega_{p} / \Omega$ as free parameters (not 'large' or 'small') in much of the analysis. Note that for weak magnetic fields, the gyroradius can still be smaller than the separation of pairs with long-range separations, so there could still be some connexion with the negative-temperature instability.

\subsection{Possible applications for three-dimensional laboratory plasmas}

The rods could represent point particles that travel freely along field lines and spread out quickly in that direction, so that all long-lived perturbations are 'flute-like' ( $\mathbf{k} \cdot \mathbf{B}=\mathbf{0})$. It has been shown that results for such three-dimensional plasmas can sometimes resemble those for two-dimensional models (cf. Lee \& Liu 1973; Cheng \& Okuda 1977).

Moreover, we tentatively suggest that our single species could represent ions rather than electrons. If so, another ion species could provide shielding, and the electrons could provide a neutralizing background. Laboratory magnetic fields being globally non-uniform, we can distinguish between ions that are reflected by 'bumps' outside the region studied, and those that pass such bumps. The untrapped particles could effectively be more mobile if there is a rotational transform $\iota$ that is not a simple fraction of $2 \pi$, and might provide shielding for the trapped particles, at least in the pertinent directions of the fluctuating electric fields (tangent to the surfaces across which important transport might occur). 
But if $\iota / 2 \pi$ is a ratio of small whole numbers, then the associated spatial periodicities need to be taken into account in the boundary conditions and the specification of the effective interparticle potential (cf. the discussion of Krommes \& Oberman (1976) between their equations (19) and (20)). Then the effective shielding could be reduced, and enhanced transport could appear, in agreement with standard conclusions based on other considerations.

Note added in proof. See Mahajan et al. (1977) and references cited there, especially Kamimura \& Dawson (1976), for somewhat similar but independently formulated ideas.

We have noted that for hybrid-time-scale behaviour to occur, the plasma must be neutrally stable if not unstable, with respect to linear instabilities. That does not rule out a stabilization at low amplitude of motion on the short-time scale, so the hybrid-time-scale behaviour is in principle observable. But it must be realized that the effects discussed by Okuda and co-workers are sometimes expected to mask at least the quantitative aspects of the effects described by the simple weak-coupling picture.

Let us note that a non-local kinetic theory of inhomogeneous three-dimensional magnetoplasma columns was previously developed (Haggerty 1967); we suggest adding periodic bumps along the magnetic field to that theory, and removing the fictitious line charges attached to the particles that in effect simplified the theory by leaving out of consideration all the evolution on the hybrid time scale. Collective effects could again be removed, in these first steps to see what can be predicted without them. See also Haggerty (1971) for the analogous gravitational kinetic theory of rotating stellar systems.

\subsection{Comparison with gravitational systems}

The effect of a uniform magnetic field on a plasma is analogous to that of the Coriolis force on a uniformly rotating gravitational system. For previous computer simulations bearing on the latter situation, we refer to Hockney (1967) and Hohl (1969). It would be interesting to see if the enhancement factor (the square root of the coupling parameter) is present in both cases.

Let us now focus on three-dimensional non-rotating gravitational systems (Haggerty \& Severne 1976), where effects have been reported that are similar to those found in the present paper.

The main difference between the two situations is in the mathematical role of the long-range cut-off $\kappa^{-1}$. There are gravitational cases where a cut-off $\kappa$ can be physically justified. For those cases, ballistic effects allow pair interactions to be completed. In the plasma case, even when $\kappa \neq 0$ there is no asymptotic separation of the unperturbed orbits; it is like a spin system.

The systematic increase in the mean kinetic energy density when $\kappa \rightarrow 0$ and $\Omega \rightarrow 0(\$ 3.3(b))$ is similar in the two cases, except that there is clustering rather than anti-clustering in the gravitational case. But one does not expect a lattice formation in the plasma; rather, an indefinite increase in the characteristic length scale. (Note also that for the three-dimensional rotating gravitational system, the similar effect pertained to motion along the axis of rotation only.) In the point-particle systems, the irreversibility was more explicit, owing to the 
scattering associated with a short-range cut-off. No coefficient $\left(t-t_{0}\right)$ appeared in equations analogous to (3.39).

The origin of the hybrid-time-scale effects in the two cases is different. For three-dimensional inhomogeneous gravitational systems it was associated with a certain commutator term that vanishes in the limit of statistical homogeneity. For (two-dimensional) plasmas we have such an essentially non-Markovian effect even with statistical homogeneity; it is now associated with the recurrences of period $2 \pi /|\Omega|$.

\section{Conclusion}

The weak-coupling kinetic equation (3.2) already yields an enhancement of evolution rates by a factor proportional to the square root of the number of rods in a Debye circle, in agreement with the findings of Hsu et al. (1974b), without the external injection of collective effects by a cut-off in the time integral. However, when the factor is very large, collective effects can become predominant.

The stroboscopic approximation gives an accelerating and eventually perhaps oscillatory relaxation towards equilibrium, via equations (3.10) and (3.22). Consistency and accuracy must be checked by computer simulation. The best tests of our version of the theory are expected to involve relatively weak magnetic fields, and relatively slowly evolving moments of the distribution functions.

In the limit of zero magnetic field, the kinetic energy density increases, and is bound only by a long-range cut-off, if present. A discussion in terms of the corresponding correlational energy density was set forth in some detail in $\$ 4$. As a by-product, we ended up with a good model to view the question of the origin of irreversibility in a novel way, avoiding the asymptotic time limit that helps to confuse the issues.

The 'quasi-stroboscopic approximation' (4.19) is suggested primarily for gyrofrequencies that are small, though larger than the inverse of the enhanced ('hybrid') time scale of evolution. It predicts non-zero evolution ((4.20), (4.22), (5.13), (5.17), (5.31)-(5.36), (5.38), (5.39), (5.41)) of certain moments that would otherwise show no secular trend, and also certain simple relationships ((5.18), $(5.20),(5.22),(5.37),(5.40))$ between these moments that are amenable to tests by computer simulation. The results suggest that for long-range vorticity, there is a mechanism for development already implicit in simple weak-coupling theory. On the other hand, effects not taken into account by such a theory, some of which have been studied in papers listed below, might often be important for quantitative evaluations of the enhanced rates of transport.

\section{REFERENCES}

Abraham-Shrauner, B. 1969 Physica, 43, 95.

BaIdwIN, D. E. \& Watson, C. J. H. 1977 Plasma Phys. 19, 517.

BAUs, M. 1977 Physica, 88A, 336; see also Phys. Rev. A 15, 790.

Belyaev, S. T. 1959 Plasma Physics and the Problem of Controlled Thermonuclear Reactions, vol. 3 (Ed. M. A. Leontovich and J. Turkevich), p. 77. Pergamon. 
Chena, C. Z. \& Okuda, H. 1977 Phys. Rev. Lett. 38, 708.

Davidson, R. \& Kozak, J. J. 1970 J. Math. Phys. 11, 1420.

EDwards, S. F. \& Taylor, J. B. 1974 Proc. Roy. Soc. A 336, 257.

Guo, T. C. \& Guo, W. W. 1975 Bull. Acad. Roy. Belgique Cl. Sc. 60, 1490; see also Physica $79 \mathrm{~A}, 120$.

HAGgerty, M. J. 1967 Phys. Fluids, 10, 2199.

Haggerty, M. J. 1971 Physica, 51, 477.

Haggerty, M. J. \& Severne, G. 1976 Adv. Chem. Phys. 35, 119.

Hinton, F. L. \& Hazeltine, R. D. 1976 Rev. Mod. Phys. 48, 239.

HockNEY, R. W. 1967 Astrophys. J. 150, 797.

HонL, F. 1969 NASA Technical Note D-5200.

Hst, J..Y., Montqomery, D. \& Joyce, G. 1974 a J. Plasma Phys. 12, 21.

Hsv, J.-Y., Joyce, G. \& MonTaomery, D. 1974 b J. Plasma Phys. 12, 27.

ICHIMARU, S. 1973 Basic Principles of Plasma Physics. Benjamin.

ICHMARU, S. \& Rosenbluth, M. N. 1970 Phys. Fluids, 13, 2778.

Joyce, G. \& MonTgomery, D. 1973 J. Plasma Phys. $10,107$.

Kamimura, T. \& Dawson, J. M. 1976 Phys. Rev. Lett. 36, 313.

Krommes, J. A. \& Oberman, C. 1976 J. Plasma Phys. 16, 229.

LeE, Y. C. \& LrU, C. S. 1973 Phys. Rev. Lett. 30, 361.

Mafajan, S. M., Guzdar, P. N., Kaw, P. K. \& Oberman, C. 1977 Princeton Plasma Physics Laboratory Preprint 1351.

MrNorsKY, N. 1962 Nonlinear Oscillations, ch. 16. Van Nostrand.

Mrsauich, J. H. \& Balescu, R. $1975 a$ Physica, 79 C, 373.

Misquich, J. H. \& Balesco, R. 1975 b Bull. Acad. Roy. Belgique Cl. Sc. 61, 210.

Moffat, M. J. 1974 Physica, 74, 355.

Montgomery, D. 1967 Lectures in Theoretical Physics, vol. $9 \mathrm{C}$ (Ed. W. E. Brittin). Gordon and Breach.

Montgomery, D. 1975 Plasma Physics: Les Houches 1972 (Ed. C. DeWitt and J. Peyraud). Gordon and Breach.

Montgomery, D. 1976 Physica, $82 \mathrm{C}, 111$.

Okuda, H. \& Dawson, J. 1973 Phys. Fluids, 16, 408.

Ostriker, J. P. \& Davidsen, A. F. 1968 Astrophys. J. $151,679$.

RADIN, S. 1972 Phys. Fluids, 15, 91.

RAdIN, S., KrITZ, A. H. \& SANDRI, G. 1967 Phys. Rev. 157, 150.

RAE, J. 1973 Recent Advances in Dynamical Astronomy (Ed. B. D. Tapley and V. Szebehely), p. 262. Reidel.

RaE, J. \& Davidson, R. 1973 J. Math. Phys. 14, 1706.

Rosenbluth, M. \& Ichimaru, S. 1972 Phys. Fluids, 15, 956.

Sagdeev, R. Z. \& Galeev, A. A. 1969 Nonlinear Plasma Theory. Benjamin.

SANDRI, G. 1963 Phys. Rev. Lett. 11, 178.

Surn, V. P. 1963 Soviet Phys. JETP, 16, 1281 ; see also Radiofisika, 6, 702.

Silin, V. P. \& Cherny, G. P. 1969 Sov. Phys. Tech. Phys. 14, 589.

Taylor, J. B. \& MoNamara, B. 1971 Phys. Fluids, 14, 1492.

VAHALA, G. $1972 J$. Plasma Phys. 8, 357.

VaHALA, G. \& Montgomery, D. 1971 J. Plasma Phys. 6, 425.

Voslamber, D. 1972 Phys. Fluids, 15, 955.

Wensstock, J. 1976 Phys. Fluids, 19, 531. 